\title{
VẤN ĐỀ LÀM KHỚP CÁC GIÁ TRI DỊ THƯỜNG ĐỘ CAO TRỌNG LỰC CỤC BỘ VÀ GPS/THỦY CHUẢN TRONG BÀI TOÁN HIỆN ĐẠ I HÓA HỆ ĐỘ CAO QUỐC GIA
}

\author{
PGS. TSKH. HÀ MINH HÒA \\ Viện Khoa học Đo đạc và Bản đồ
}

\section{Tóm tắt:}

Bài báo khoa học này xác định tiêu chí lựa chọn các điểm độ cao hạng I, II có các giá trị tuyệt đối của các hiệu giữa dị thường độ cao GPS/thủy chuẩn và dị thường độ cao trọng lực cục bộ nhỏ hơn $10 \mathrm{~cm}$ để xây dựng mô hình quasigeoid quốc gia cuối cùng trên cơ sở làm khớp các giá trị dị thường độ cao GPS/thủy chuẩn với các giá trị dị thường độ cao trọng lực cục bộ theo phương pháp collocation trung phương. Ngoài ra, bài báo còn đề xuất phương pháp làm khớp các giá trị dị thường độ cao nêu trên bằng cách hiệu chỉnh các hệ số khai triển điều hòa cầu của mô hình EGM2008 dựa trên các thế nhiễu trên các điểm co' sở hạng $B$.

\section{1. Đặt vấn đề}

Như đã chỉ ra trong tài liệu (Hà Minh Hòa (2013a)), cơ sở khoa học nền tảng để xây dựng hệ quy chiếu tọa độ không gian quốc gia ở Việt Nam là mô hình quasigeoid quốc gia độ chính xác cao. Mặt khác, hệ độ cao quốc gia ở Việt Nam là hệ độ cao chuẩn dựa trên mặt quasigeoid quốc gia. Do đó việc xây dựng mô hình quasigeoid quốc gia độ chính xác cao không chỉ tạo cơ sở cho việc xây dựng hệ quy chiếu tọa độ không gian quốc gia, mà còn dẫn đến việc hoàn thiện và hiện đại hóa hệ độ cao quốc gia.

Trong các tài liệu (Hà Minh Hòa (2014b); Hà Minh Hòa (2014c); Hà Minh Hòa (2014d); Hà Minh Hòa (2014f)) đã trình bày công thức xác định dị thường độ cao trọng lực cục bộ trong mô hình quasigeoid quốc gia VIGAC2014, theo đó dị thường độ cao trọng lực cục bộ $\hat{\bar{\zeta}}_{z}$ trong hệ triều 0 được chuyển từ mô hình EGM2008 tương ứng với mặt quasigeoid toàn cầu về mặt quasigeoid cục bộ Hòn Dấu theo công thức:

$$
\hat{\bar{\zeta}}_{z}=\bar{\zeta}+0,890 m+\delta \zeta_{n-z},
$$

đây $\bar{\zeta}$ - dị thường độ cao toàn cầu từ mô hình trọng trường Quả đất EGM2008 tương ứng với mặt quasigeoid toàn cầu trong hệ không phụ thuộc triều, $\delta \zeta_{n-z}$ - số cải chính chuyển dị thường độ cao từ hệ không phụ thuộc triều về hệ triều 0 và có dạng:

$$
\delta \zeta_{n-z}=\left(0,02871-0,08584 \cdot \sin ^{2} B\right)<m>.
$$

Trong các tài liệu nêu trên đã giới thiệu các kết quả đánh giá mô hình quasigeoid quốc gia VIGAC2014 dựa trên 09 điểm cơ sở là các điểm độ cao hạng I (chúng ta gọi các điểm này

Người phản biện: TS. Nguyễn Đình Thành 
là các điểm cơ sở hạng $A$ vì chúng thực sự tương ứng với mặt Quasigeoid VIGAC2014) bao gồm các điểm I(HN-VL)6-1, I(HN-VL)28-1, I(HN-VL)64, I(HN-VL)72, I(VL-HT)98, I(VL$\mathrm{HT})$ 158, I(BH-HN)33, I(BH-TH)65, I(BH-TH)122A, ở đây các dị thường độ cao của điểm độ cao hạng I này thực sự tương ứng với mô hình quasigeoid quốc gia VIGAC2014 với sai số trung phương ở mức $\pm 5 \mathrm{~mm}$ (xem trong các tài liệu Hà Minh Hòa (2014b); Hà Minh Hòa (2014f)). Các kết quả xác định các giá trị độ cao chuẩn trên các điểm GPS được bố trí trên các đảo Phú Quốc, Côn Đảo (xem trong tài liệu Hà Minh Hòa (2014d)) và ở khu vực rừng núi bao gồm 04 điểm C004 (Điện Biên), C022 (Sơn La), C065 (Tuyên Quang) và C075 (Quảng Ninh) thuộc mạng lưới trắc địa địa động lực miền Bắc được đo liên tục trong 4 ngày đêm trong tháng 01/2013 (xem trong tài liệu Hà Minh Hòa (2014f)) dựa trên mô hình quasigeoid quốc gia VIGAC2014 và được truyền từ 09 điểm cơ sở hạng A cho thấy các độ cao chuẩn được xác định bằng hai phương pháp nêu trên chỉ chênh nhau có $2 \mathrm{~mm}$.

Sự chênh nhau nhỏ bỏ qua giữa các giá trị độ cao chuẩn của các điểm GPS được xác định từ mô hình quasigeoid quốc gia VIGAC2014 và được truyền từ 09 điểm cơ sở hạng $\mathrm{A}$ cho thấy rằng các sai số bị bỏ qua (ommision errors) trong dị thường độ cao trọng lực $\zeta$ được xác định từ mô hình trọng trường Quả đất EGM2008 trong công thức (1) là các đại lượng không đổi trên lãnh thổ Việt Nam và bị loại bỏ khi xác định dị thường độ cao trọng lực cục bộ theo công thức (1) (xem chứng minh trong tài liệu Hà Minh Hòa (2014f)).

Trong tài liệu (Hirt C., Featherstone W. E., Marti U. (2010)) đã thông báo rằng sai số bị bỏ qua của dị thường độ cao trọng lực được xác định từ mô hình EGM2008 có thể đạt tới $10 \mathrm{~cm}$ ở vùng núi. Khi sử dụng mô hình RTM (Residual Terrain Model), độ cao được xây dựng như hiệu của SRTM và DTM2006.0 sẽ nâng độ chính xác xác định dị thường độ cao trọng lực đến $1,9 \mathrm{~cm}$. Việc sử dụng RTM độ cao sẽ nâng cao độ chính xác xác định dị thường độ cao trọng lực ở các khu vực rừng núi ở Châu Á, Nam Mỹ và Châu Phi mà không cần đến dữ liệu trọng lực khu vực.

Như vậy, các kết quả thực nghiệm ở Việt Nam và các thực tiễn trên thế giới xác định các cơ sở để khẳng định rằng mô hình quasigeoid quốc gia VIGAC2014 là mô hình quasigeoid độ chính xác cao ở Việt Nam.

Tuy nhiên, khi đã có mô hình quasigeoid độ chính xác cao, chúng ta không thể có ngay hệ độ cao quốc gia hiện đại với các độ cao chuẩn của các điểm độ cao hạng I, II quốc gia chính xác và đồng nhất trên toàn bộ lãnh thổ quốc gia. Như đã trình bày trong tài liệu (Hà Minh Hòa (2014c)), hệ độ cao quốc gia ở Việt Nam cũng như các hệ độ cao ở các nước khác có lịch sử xây dựng và khai thác nhiều thập kỷ. Dưới tác động của các hiện tượng tự nhiên như chuyển dịch đứng của vỏ Trái đất, sự thay đổi của mực nước ngầm v...v và tác động của các hoạt động kinh tế - xã hội của con người như khai thác mỏ làm thay đổi bề mặt Trái đất, xây dựng các hồ chứa nước, bạt núi, lấp biển và các công trình lớn làm thay đổi cục bộ trọng trường của khu vực, xây dựng và mở rộng các đường giao thông, xây dựng các khu đô thị lớn v...v làm các mốc độ cao quốc gia bị xê dịch. Điều này dẫn đến sự thay đổi lớn của các giá trị độ cao của các mốc độ cao. Trong tài liệu (Hà Minh Hòa (2014c)) đã đưa ra đánh giá các độ chênh của dị thường độ cao trọng lực cục bộ và dị thường độ cao GPS/thủy chuẩn trên 230 điểm độ cao hạng I ở Việt Nam, theo đó chỉ có 156 điểm độ cao hạng I (chiếm 67.83\%) có các độ chênh thỏa mãn hạn sai.

Chính vì sự thay đổi độ cao của nhiều mốc độ cao quốc gia trải qua nhiều thập kỷ xây dựng và khai thác, không một quốc gia nào sẵn sàng bỏ ra kinh phí rất lớn và mất nhiều 
thập kỷ nữa để đo lại mạng lưới độ cao quốc gia. Đất nước rộng lớn như Canada quyết định loại bỏ hoàn toàn hệ độ cao cũ CGVD 28 và xây dựng hệ độ cao mới CGVD2013 dựa trên mô hình geoid CGG2013 và công nghệ GPS (Veronneau M., Huang J. (2007); Véronneau M. (2014)). Vương quốc Anh có diện tích không lớn hơn Việt Nam bao nhiêu quyết định sử dụng lại hệ độ cao cũ ODN (Ordnance Datum Newlyn) của nước Anh, nhưng chỉ sử dụng 179 điểm độ cao ổn định nhất có các độ chênh nhỏ nhất giữa độ cao geoid GPS/thủy chuẩn và độ cao geoid trọng lực được xác định từ mô hình geoid trọng lực mới OSGM02 để hoàn thiện hệ độ cao ODN, theo đó các giá trị độ cao geoid GPS/thủy chuẩn và độ cao geoid trọng lực trên 179 điểm này được làm khớp theo phương pháp callocation trung phương để hoàn thiện mô hình Geoid OSGM02. Các độ cao chính của các điểm độ cao quốc gia còn lại trong hệ ODN được tính toán lại dựa trên mô hình Geoid OSGM02 và công nghệ GPS (Forsberg R., Strykowski G., lliffe J.C., Ziebart M., Cross P.A., Tscherning C.C., Cruddace P. (2001); lliffe J.C., Ziebart M., Cross P.A., Forsberg R., Strykowski G., Tscherning C.C. (2003)).

Trong tài liệu (Hà Minh Hòa (2014f)) đã định hướng hoàn thiện hệ độ cao HN72 ở Việt Nam theo phương án của Vương quốc Anh. Các vấn đề khoa học cần giải quyết bao gồm:

- Xác định tiêu chí để lựa chọn các điểm độ cao hạng I, II quốc gia mà các giá trị dị thường độ cao GPS/thủy chuẩn của chúng được sử dụng để làm khớp với các giá trị dị thường độ cao trọng lực cục bộ tương ứng trên các điểm này (chúng ta gọi các điểm độ cao hạng I, II được chọn là các điểm cơ sở hạng $\mathrm{B}$ );

- Phương pháp làm khớp các giá trị dị thường độ cao GPS/thủy chuẩn với các giá trị dị thường độ cao trọng lực cục bộ của các điểm độ cao quốc gia được lựa chọn.

Bài báo khoa học này sẽ giải quyết các vấn đề khoa học nêu trên.

\section{Giải quyết vấn đề}

\subsection{Xác định tiêu chí lực chọn các điểm cơ sở hạng $B$}

Mô hình quasigeoid trọng lực cục bộ được xác định theo công thức (1) được gọi là mô hình quasigeoid quốc gia khởi đầu. Sau khi làm khớp các giá trị dị thường độ cao GPS/thủy chuẩn với các giá trị dị thường độ cao trọng lực cục bộ của $\mathrm{N}$ điểm cơ sở hạng $\mathrm{B}$, chúng ta sẽ nhận được mô hình quasigeoid quốc gia cuối cùng.

Khi đo GPS trên các điểm độ cao hạng I,II quốc gia và xử lý các dữ liệu GPS trong ITRF tương ứng với ellipsoid WGS84 quốc tế, dị thường độ cao GPS/thủy chuẩn $\zeta_{z}$ trong hệ triều 0 (zero - tide system) của điểm độ cao quốc gia bất kỳ được xác định theo công thức:

$$
\zeta_{z}=\bar{H}_{z}-H_{z}^{\gamma},
$$

Ở đây $\bar{x}_{z}$ - độ cao trắc địa của điểm tương ứng với ellipsoid WGS84 quốc tế trong hệ triều 0 ; $g_{g}^{\prime}$ - độ cao chuẩn của điểm trong hệ triều 0 .

Dị thường độ cao GPS/thủy chuẩn $\zeta_{z}$ (2) hoàn toàn độc lập với dị thường độ cao trọng lực $\xi_{\dot{z}}$ (1). Các giá trị dị thường độ cao GPS/thủy chuẩn là nguồn dữ liệu quan trọng để làm chính xác mô hình quasigeoid trọng lực cục bộ được xác định theo công thức (1) dựa trên phương pháp collocation trung phương. Giả thiết rằng trên toàn bộ các điểm độ cao hạng I, II quốc gia chúng ta đã xác định được $\mathrm{N}$ điểm độ cao là các điểm cơ sở hạng $\mathrm{B}$. 


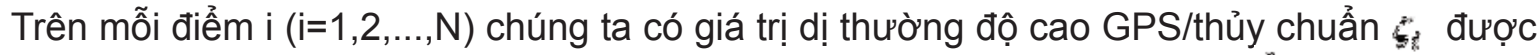
xác định theo công thức (2) và giá trị dị thường độ cao trọng lực cục bộ được xác định theo công thức (1). Đặt $d_{i}=\zeta_{i}-\hat{\zeta}_{i}$ là hiệu của giá trị dị thường độ cao GPS/thủy chuẩn và giá trị dị thường độ cao trọng lực cục bộ. Khi ký hiệu tớ là giá trị dị thường độ cao có độ chính xác cao tương ứng với mô hình quasigeoid quốc gia cuối cùng, chúng ta lập hai phương trình số cải chính:

$$
\begin{aligned}
& \delta \hat{\bar{\zeta}}_{i}=\widetilde{\zeta}_{i}-\hat{\zeta}_{i}, \\
& \delta \zeta_{i}=\widetilde{\zeta}_{i}-\zeta_{i},
\end{aligned}
$$

ở đây $\delta \hat{\bar{\zeta}}_{i}$ - số cải chính vào giá trị dị thường độ cao trọng lực cục bộ 颜 để nhận được giá trị $\widetilde{\zeta}_{i}$ còn $\delta \zeta_{i}$ - số cải chính vào giá trị dị thường độ cao GPS/thủy chuẩn để đhận được giá trị 落

Khi giải hệ (3) dưới điều kiện $\delta \hat{\bar{\zeta}}_{i}^{2}+\delta \zeta_{i}^{2}=$ min, chúng ta nhận được:

$$
\widetilde{\zeta}_{i}=\frac{\hat{\bar{\zeta}}_{i}+\zeta_{i}}{2} \text {. }
$$

Như vậy, giá trị dị thường độ cao $Z_{\sharp}^{Z}$ có độ chính xác cao trên điểm i nhận được bằng cách lấy trung bình các giá trị dị thường độ cao GPS/thủy chuẩn trọng lực cục bộ $\hat{\bar{\zeta}}_{\mathrm{H}}$. Khi lưu ý (4), số cải chính $\delta \hat{\bar{\zeta}}_{i}$ từ phương trình thứ nhất của hệ (3) có dạng:

$$
\delta \hat{\bar{\zeta}}_{i}=\frac{\zeta_{i}-\hat{\bar{\zeta}}_{i}}{2}=\frac{d_{i}}{2} .
$$

Trong trường hợp làm khớp (fitting) các giá trị dị thường độ cao GPS/thủy chuẩn và dị thường độ cao trọng lực cục bộ trên $\mathrm{N}$ điểm cơ sở hạng $\mathrm{B}$ để làm chính xác mô hình quasigeoid quốc gia khởi đầu theo phương pháp collocation trung phương, vectơ các số cải chính $\delta \hat{\bar{\zeta}}$ được xác định theo công thức:

ở đây d - vectơ các hiệu $d_{i}$ nêu ở trên.

$$
\delta \hat{\bar{\zeta}}=C_{\delta \hat{\bar{\zeta}}, d} .\left(C_{d}+D_{d}\right)^{-1} \cdot d,
$$

Trong thực tế, công thức (6) được sử dụng để xác định các số cải chính vào các giá trị dị thường độ cao trọng lực trên các điểm nút của các ô chuẩn (grid) dị thường độ cao cục bộ để nhận được mô hình quasigeoid quốc gia cuối cùng. Do đó, độ chính xác của các giá trị cuối cùng của các dị thường độ cao trên $\mathrm{N}$ điểm cơ sở hạng $\mathrm{B}$ sẽ đặc trưng cho độ chính xác của mô hình quasigeoid quốc gia cuối cùng. Chúng ta xem xét việc đánh giá độ chính xác của giá trị cuối cùng của dị thường độ cao trên $\mathrm{N}$ điểm cơ sở hạng $\mathrm{B}$ theo phương pháp collocation trung phương khi bỏ qua các mối liên hệ không gian giữa các điểm này. Với điều kiện này, đối với mỗi điểm i thuộc tập hợp $\mathrm{N}$ điểm cơ sở hạng $\mathrm{B}$ chúng ta có thể nhận $C_{\delta \hat{\bar{\delta}}, d}=C_{d}=D_{d}=C_{0}$. Khi đó từ (6) đối với điểm i chúng ta lại nhận được công thức (5). Như vậy, chúng ta có thể coi giá trị trung bình (4) là giá trị dị thường độ cao có độ chính xác cao nhất trên điểm i thuộc tập hợp $\mathrm{N}$ điểm cơ sở hạng $\mathrm{B}$. Chúng ta hoàn toàn có cơ sở để coi các hiệu $d_{i}=\zeta_{i}-\hat{\bar{\zeta}}_{i}$ có sai số trung phương như nhau. Khi đó, độ chính xác của 
mô hình quasigeoid quốc gia cuối cùng được đánh giá theo công thức:

$$
m_{\tilde{\zeta}}= \pm \frac{1}{2} \cdot \sqrt{\frac{\sum_{i=1}^{N} d_{i}^{2}}{N}} .
$$

Để xác định tiêu chí lựa chọn các điểm độ cao hạng I, II thuộc tập hợp $\mathrm{N}$ điểm cơ sở hạng $\mathrm{B}$ từ toàn bộ các điểm thuộc mạng lưới độ cao hạng $\mathrm{I}$, II quốc gia với giả thiết rằng trên tất cả các điểm độ cao hạng I, II quốc gia có đo GPS và xử lý các dữ liệu GPS trong ITRF, chúng ta cần xác định giá trị cực đại $\mathrm{d}_{\max }$ cho phép của các hiệu $d_{i}=\zeta_{i}-\hat{\bar{\zeta}}_{i}$ sao cho sai số trung phương maz $_{z}$ thỏa mãn yêu cầu độ chính xác của mô hình quasigeoid quốc gia cuối cùng. Khi ký hiệu $m_{g \cdot h}=2,5 \cdot m_{\widetilde{\zeta}}$ là sai số trung phương giới hạn của dị thường độ cao tương ứng với mô hình quasigeoid quốc gia cuối cùng, từ (7) chúng ta có điều kiện:

$$
m_{g h}=2,5 \cdot m_{\widetilde{\zeta}} \geq \frac{1}{2} \cdot \sqrt{\frac{\sum_{i=1}^{N} d_{i}^{2}}{N} .}
$$

Khi coi $d_{1}=d_{2}=\ldots=d_{N}=d_{\max }$, từ bất đẳng thức trên suy ra $d_{\max } \leq 5 \cdot m_{\widetilde{\zeta}}$.

Nếu độ chính xác của mô hình quasigeoid quốc gia cuối cùng đạt $m_{\widetilde{\zeta}}= \pm 2 \mathrm{~cm}$, thì $\mathrm{d}_{\max }$ $\leq 10 \mathrm{~cm}=1 \mathrm{dm}$. Như vậy, tiêu chí lựa chọn các điểm độ cao hạng I, II thuộc tập hợp $\mathrm{N}$ điểm cơ sở hạng $\mathrm{B}$ là: Giá trị tuyệt đối của các hiệu $d_{i}=\zeta_{i}-\hat{\bar{\zeta}}_{i} \quad(\mathrm{i}=1,2, \ldots, \mathrm{N})$ không được lớn hơn $10 \mathrm{~cm}$. Dựa trên tiêu chí này, từ 230 điểm độ cao hạng I có đo GPS và xử lý các dữ liệu GPS trong ITRF, chúng ta chọn được 85 điểm độ cao hạng I, trong đó 09 điểm thuộc các điểm cơ sở hạng $A$ có các giá trị dị thường độ cao trung bình (4) thực sự đã thuộc mô hình quasigeoid quốc gia cuối cùng và được sử dụng để đánh giá mô hình quasigeoid quốc gia khởi đầu VIGAC2014, còn 76 điểm độ cao hạng I thuộc các điểm cơ sở hạng B. Các kết quả đánh giá độ chính xác của mô hình quasigeoid quốc gia cuối cùng theo công thức (7) dựa trên 76 điểm cơ sở hạng $B$ được trình bày ở bảng dưới đây. (Xem bảng 1)

$$
m_{\widetilde{\zeta}}= \pm \frac{1}{2} \cdot \sqrt{\frac{\sum_{i=1}^{N} d_{i}^{2}}{N}}= \pm \frac{1}{2} \cdot \sqrt{\frac{0,126364}{76}}= \pm 2,0 \mathrm{~cm} .
$$

Các phân tích lý thuyết và thực nghiệm trên các điểm độ cao hạng I ở Việt Nam cho thấy rằng với mục đích xây dựng mô hình quasigeoid quốc gia cuối cùng với độ chính xác ở mức $\pm 2 \mathrm{~cm}$, chúng ta xác định tập hợp các điểm cơ sở hạng $B$ mà tại đó các giá trị tuyệt đối của các độ chênh giữa dị thường độ cao GPS/thủy chuẩn (2) và dị thường độ cao trọng lực cục bộ (1) không được vượt quá $10 \mathrm{~cm}$.

\subsection{Phương pháp làm khớp dị thường độ cao GPS/thủy chuẩn và dị thường độ cao trọng lực cục bộ}

Như đã trình bày ở trên, phương pháp phổ biến để làm khớp dị thường độ cao GPS/thủy chuẩn và dị thường độ cao trọng lực cục bộ trong bài toán xây dựng mô hình quasigeoid quốc gia cuối cùng là phương pháp collocation trung phương. Tuy nhiên, trong bài toán rộng lớn hơn - bài toán xây dựng mô hình trọng trường Trái đất phù hợp với trọng 
Bảng 1: Đánh giá độ chính xác của dị thường độ cao trung bình trên các điểm cơ sở hạng $B$

\begin{tabular}{|c|c|c|c|c|c|}
\hline STT i & Tên điểm & $\begin{array}{c}\text { Hiệu } \\
d_{i}=\left(\zeta_{i}\right)_{z}-\left(\hat{\bar{\zeta}}_{i}\right)_{z} \\
(\mathrm{~m})\end{array}$ & STT i & Tên điểm & $\begin{array}{c}\text { Hiệu } \\
d_{i}=\left(\zeta_{i}\right)_{z}-\left(\hat{\zeta}_{i}\right)_{z} \\
\text { (m) }\end{array}$ \\
\hline \multicolumn{6}{|c|}{ Các kết quả đo của Cục Đo đạc và Bản đồ Việt Nam giai đoạn 2009 - 2010} \\
\hline 1 & $\mathrm{I}(\mathrm{BH}-\mathrm{LS}) 97$ & 0,093 & 17 & $\mathrm{I}(\mathrm{BH}-\mathrm{TH}) 5$ & $-0,038$ \\
\hline 2 & $\mathrm{I}(\mathrm{BH}-\mathrm{TH}) 122 \mathrm{~A}$ & 0,006 & 18 & $\mathrm{I}(\mathrm{VL}-\mathrm{HT}) 141-3$ & $-0,014$ \\
\hline 3 & $\mathrm{I}(\mathrm{HN}-\mathrm{VL}) 10 \mathrm{~A}$ & $-0,093$ & 19 & $\mathrm{I}(\mathrm{BH}-\mathrm{TH}) 70 \mathrm{~A}$ & 0,075 \\
\hline 4 & $\mathrm{I}(\mathrm{BH}-\mathrm{TH}) 119$ & 0,026 & 20 & $\mathrm{I}(\mathrm{HN}-\mathrm{VL}) 50$ & 0,070 \\
\hline 5 & $\mathrm{I}(\mathrm{BH}-\mathrm{HN}) 33$ & 0,009 & 21 & $\mathrm{I}(\mathrm{VL}-\mathrm{HT}) 123$ & 0,064 \\
\hline 6 & $\mathrm{I}(\mathrm{BH}-\mathrm{HN}) 39$ & 0,014 & 22 & $\mathrm{I}(\mathrm{LS}-\mathrm{HN}) 12$ & 0,079 \\
\hline 7 & $\mathrm{I}(\mathrm{BH}-\mathrm{HN}) 42$ & $-0,014$ & 23 & I(HN-VL)38-1 & $-0,042$ \\
\hline 8 & $\mathrm{I}(\mathrm{HN}-\mathrm{HP}) 7$ & 0,059 & 24 & I(DN-BT)86 & 0,069 \\
\hline 9 & $\mathrm{I}(\mathrm{HN}-\mathrm{VL}) 4-1$ & 0,023 & 25 & $\mathrm{I}(\mathrm{VL}-\mathrm{HT}) 320 \mathrm{~A}$ & 0,067 \\
\hline 10 & $\mathrm{I}(\mathrm{HN}-\mathrm{VL}) 6-1$ & $-0,006$ & 26 & I(HP-C)4-1 & 0,085 \\
\hline 11 & $\mathrm{I}(\mathrm{DN}-\mathrm{BT}) 16$ & $-0,097$ & 27 & I(BH-TH)3-1 & $-0,044$ \\
\hline 12 & I(DN-BT)28 & $-0,091$ & 28 & $\mathrm{I}(\mathrm{VL}-\mathrm{HT}) 181$ & $-0,084$ \\
\hline 13 & $\mathrm{I}(\mathrm{VL}-\mathrm{HT}) 150$ & $-0,095$ & 29 & I(LS-TY)4 & $-0,060$ \\
\hline 14 & $\mathrm{I}(\mathrm{VL}-\mathrm{HT}) 152-1$ & $-0,088$ & 30 & $\mathrm{I}(\mathrm{VL}-\mathrm{HT}) 309 \mathrm{~A}$ & $-0,081$ \\
\hline 15 & $\mathrm{I}(\mathrm{HN}-\mathrm{VL}) 34-1$ & $-0,072$ & 31 & $\mathrm{I}(\mathrm{VL}-\mathrm{HT}) 317$ & $-0,076$ \\
\hline 16 & I(HP-MC)48A & $-0,068$ & 32 & $\mathrm{I}(\mathrm{VL}-\mathrm{HT}) 187$ & $-0,072$ \\
\hline & & & & & \\
\hline 33 & $\mathrm{I}(\mathrm{VL}-\mathrm{HT}) 170-1$ & $-0,071$ & 55 & I(DN-BT)83 & 0,029 \\
\hline 34 & I(HP-MC)41 & $-0,042$ & 56 & $\mathrm{I}(\mathrm{VL}-\mathrm{HT}) 78$ & 0,032 \\
\hline 35 & $\mathrm{I}(\mathrm{VL}-\mathrm{HT}) 130$ & $-0,058$ & 57 & $\mathrm{I}(\mathrm{LS}-\mathrm{HN}) 7$ & 0,055 \\
\hline 36 & $\mathrm{I}(\mathrm{HN}-\mathrm{VL}) 56$ & 0,028 & 58 & $\mathrm{I}(\mathrm{VL}-\mathrm{HT}) 71$ & 0,051 \\
\hline 37 & $\mathrm{I}(\mathrm{BH}-\mathrm{TH}) 11$ & 0,041 & 59 & $\mathrm{I}(\mathrm{BH}-\mathrm{TH}) 59$ & 0,074 \\
\hline 38 & I(HN-VL)40-1 & 0,034 & 60 & I(VL-HT)173-2 & $-0,056$ \\
\hline 39 & I(BH-LS)77 & 0,043 & 61 & I(VL-HT)197 & $-0,055$ \\
\hline 40 & $\mathrm{I}(\mathrm{VL}-\mathrm{HT}) 329 \mathrm{~A}$ & $-0,014$ & 62 & I(BT-APD)63 & $-0,055$ \\
\hline 41 & $\mathrm{I}(\mathrm{HN}-\mathrm{VL}) 72$ & 0,001 & 63 & I(VL-HT)127-3 & $-0,049$ \\
\hline 42 & I(VL-HT)158 & 0,000 & 64 & I(BT-APD)59-1 & $-0,052$ \\
\hline 43 & $\mathrm{I}(\mathrm{VL}-\mathrm{HT}) 121$ & 0,007 & 65 & I(VL-HT)278-1 & $-0,046$ \\
\hline 44 & $\mathrm{I}(\mathrm{DN}-\mathrm{BT}) 74$ & 0,022 & 66 & $\mathrm{I}(\mathrm{VL}-\mathrm{HT}) 108$ & $-0,038$ \\
\hline 45 & $\mathrm{I}(\mathrm{BH}-\mathrm{LS}) 88-1$ & 0,024 & 67 & I(DN-BT)77 & $-0,035$ \\
\hline 46 & I(VL-HT)98 & 0,009 & 68 & I(BT-NH)17-1 & $-0,038$ \\
\hline 47 & $\mathrm{I}(\mathrm{BH}-\mathrm{LS}) 85-1$ & 0,028 & 69 & $\mathrm{I}(\mathrm{BH}-\mathrm{LS}) 80$ & 0,087 \\
\hline 48 & I(BH-LS)93 & 0,026 & 70 & $\mathrm{I}(\mathrm{VL}-\mathrm{HT}) 83$ & $-0,032$ \\
\hline 49 & $\mathrm{I}(\mathrm{BH}-\mathrm{LS}) 71$ & 0,031 & 71 & $\mathrm{I}(\mathrm{BH}-\mathrm{HN}) 17$ & $-0,017$ \\
\hline 50 & I(BT-APD)56 & 0,011 & 72 & $\mathrm{I}(\mathrm{HN}-\mathrm{VL}) 45-1$ & $-0,022$ \\
\hline 51 & $\mathrm{I}(\mathrm{VL}-\mathrm{HT}) 87$ & 0,028 & 73 & I(BH-TH)65 & $-0,008$ \\
\hline 52 & I(VL-HT)247A & 0,022 & 74 & $\mathrm{I}(\mathrm{VL}-\mathrm{HT}) 178$ & $-0,022$ \\
\hline 53 & I(LS-TY)1 & 0,042 & 75 & I(VL-HT)103 & $-0,015$ \\
\hline 54 & $\mathrm{I}(\mathrm{VL}-\mathrm{HT}) 325-1$ & 0,023 & 76 & $\mathrm{I}(\mathrm{HN}-\mathrm{VL}) 64$ & $-0,006$ \\
\hline
\end{tabular}


trường trên lãnh thổ quốc gia, chúng ta phải áp dụng cách tiếp cận khác - hiệu chỉnh các hệ số khai triển điều hòa của mô hình trọng trường Trái đất EGM2008 cho phù hợp với trọng trường trên lãnh thổ quốc gia. Trong tài liệu (Hà Minh Hòa, Nguyễn Tuấn Anh (2014e)) đã giới thiệu các kết quả thực nghiệm hiệu chỉnh các hệ số khai triển điều hòa của mô hình trọng trường Trái đất EGM2008 từ mức 181 đến mức 2190 dựa trên các ô chuẩn dị thường trọng lực Faye $5^{\prime}$ x 5' nằm ở phía Bắc Việt Nam trong phạm vi $\mathrm{B}_{\text {min }}=$ $20^{\circ}, 3 ; B_{\max }=21^{\circ}, 5 ; L_{\min }=105^{\circ}, 1 ; L_{\max }=106^{\circ}, 3$. Các kết quả thực nghiệm cho thấy các giá trị dị thường trọng lực Faye được xác định từ mô hình trọng trường Trái đất EGM2008 được hiệu chỉnh sát nhất với các giá trị thực của các dị thường trọng lực Faye được xác định từ các kết quả đo đạc trọng lực chi tiết. Do các giá trị độ cao chuẩn $H^{\gamma}$ và độ cao trắc địa $\mathrm{H}$ (được xác định bằng công nghệ GPS) hoàn toàn độc lập với các dữ liệu trọng lực chi tiết, nên trong các tài liệu (Hà Minh Hòa (2014a); Hà Minh Hòa (2014b)) đã đề xuất sử dụng các giá trị thế nhiễu được xác định từ các giá trị độ cao chuẩn $H^{\gamma}$ và độ cao trắc địa $\mathrm{H}$ trên các điểm độ cao hạng I, II quốc gia thuộc tập hợp các điểm cơ sở hạng $B$ để hiệu chỉnh các hệ số khai triển điều hòa của mô hình trọng trường Trái đất EGM2008. Khi đó, các giá trị dị thường độ cao trọng lực cục bộ được xác định từ mô hình trọng trường Trái đất EGM2008 được hiệu chỉnh sẽ phù hợp với mô hình quasigeoid quốc gia cuối cùng. Trong trường hợp này, do các giá trị độ cao chuẩn $H^{\gamma}$ và độ cao trắc địa $\mathrm{H}$ trên các điểm độ cao hạng I, II quốc gia thuộc tập hợp các điểm cơ sở hạng $B$ đã được sử dụng, nên không còn ý nghĩa xác định các giá trị dị thường độ cao GPS/thủy chuẩn theo công thức (2).

Tiền đề cho việc giải quyết bài toán nêu trên là xác định được thế trọng trường thực $\mathrm{W}_{0}$ $=62636847,2911 \mathrm{~m}^{2} \cdot \mathrm{s}^{-2}$ của mặt geoid cục bộ sát nhất với mặt biển trung bình tại trạm nghiệm triều Hòn Dấu với sai số trung phương $\pm 0,183 \mathrm{~m}^{2} \cdot \mathrm{s}^{-2}$ (xem các tài liệu Hà Minh Hòa (2012); Hà Minh Hòa (2013b); Hà Minh Hòa (2014b)). Khi đó, thế trọng trường thực (đơn vị $\mathrm{m}^{2} \cdot \mathrm{s}^{-2}$ ) của điểm độ cao i bất kỳ thuộc tập hợp các điểm cơ sở hạng $\mathrm{B}$ được xác định theo công thức:

$$
W_{i}=W_{0}-10^{-5} \cdot \bar{\gamma}_{i} \cdot H_{i}^{\gamma},
$$

ở đây $\mathrm{W}_{0}=62636847,2911 \mathrm{~m}^{2} \cdot \mathrm{s}^{-2}, \bar{y}_{i}$ - giá trị trung bình của gia tốc lực trọng trường chuẩn (đơn vị mGal) tại điểm i, $H_{i}^{\gamma}$ - độ cao chuẩn (đơn vị mét) của điểm i.

Trong khi đó, thế trọng trường chuẩn (đơn vị $\mathrm{m}^{2} \mathrm{~s}^{-2}$ ) của điểm độ cao i được xác định theo công thức:

$$
U_{i}=U_{0}-10^{-5} \cdot \bar{\gamma}_{i} \cdot H_{i},
$$

ở đây $U_{0}=62636855,812 \mathrm{~m}^{2} \cdot \mathrm{s}^{-2}$ - thế trọng trường chuẩn của ellipsoid TFS2008 với bán kính bán trục lớn $a=6378136,58 m$ và nghịch đảo độ dẹt $\frac{1}{f}=298,257686, \mathrm{H}_{i}$ - độ cao trắc địa (đơn vị mét) của điểm i.

Như vậy thế nhiễu $T_{i}=W_{i}-U_{i}$ tại điểm độ cao $i$ hoàn toàn được xác định nhờ đã biết độ cao chuẩn $H_{i}^{\gamma}$ và độ cao trắc địa $\mathrm{H}_{\mathrm{i}}$ của điểm này. Đến đây chúng ta đã thay dị thường độ cao $\zeta_{i}(2)$ bằng thế nhiễu $\mathrm{T}_{\mathrm{i}}$. Như đã chứng minh trong tài liệu (Hà Minh Hòa (2014a)), các số cải chính vào các hệ số điều hòa cầu của mô hình EGM2008 được xác định theo công thức: 


$$
\left\{\begin{array}{l}
\delta \overline{\bar{C}}_{n, m} \\
\overline{\delta \overline{\bar{S}}_{n, m}}
\end{array}\right\}=\frac{\rho}{4 \pi \cdot G \cdot M} \cdot \iint_{\sigma} \delta T(\rho, B, L) \cdot\left\{\begin{array}{c}
\cos m L \\
\sin m L
\end{array}\right\} \bar{P}_{n, m}(\sin B) \cdot d \sigma
$$

và các giá trị được hiệu chỉnh của các hệ số điều hòa cầu của mô hình trọng trường Trái đất EGM2008 được hiệu chỉnh sẽ được xác định theo công thức:

$$
\left\{\begin{array}{c}
\tilde{\overline{\bar{C}}}_{n, m} \\
\tilde{\overline{\bar{S}}}_{n, m}
\end{array}\right\}=\left\{\begin{array}{l}
\overline{\bar{C}}_{n, m} \\
\overline{\bar{S}}_{n, m}
\end{array}\right\}+\left\{\begin{array}{c}
\delta \overline{\bar{C}}_{n, m} \\
\delta \overline{\bar{S}}_{n, m}
\end{array}\right\},
$$

ở đây $G \cdot M=3,986004418.10^{14} \mathrm{~m}^{3} \cdot \mathrm{s}^{-2}$ - hằng số trọng trường địa tâm; tại mỗi điểm i với các tọa độ cầu $(\rho, B, L)$ thuộc tập hợp các điểm cơ sở hạng $\mathrm{B}$, hiệu $\delta T(\rho, B, L)$ được xác định theo công thức:

$$
\delta T(\rho, B, L)=T(\rho, B, L)-T^{E G M}(\rho, B, L),
$$

còn thế nhiễu $T^{E G M}(\rho, B, L)$ tương ứng với điểm i được xác định theo công thức:

$$
T^{E G M}(\rho, B, L)=\frac{3 \cdot 10^{5} m^{3} \cdot s^{-2}}{\rho}+\frac{G \cdot M}{\rho} \cdot \sum_{n=2}^{\infty} \sum_{m=0}^{n}\left(\overline{\bar{C}}_{n, m} \cdot \cos m L+\overline{\bar{S}}_{n, m} \cdot \sin m L\right) \bar{P}_{n, m}(\sin B),
$$

với ký hiệu $\beta=1$ : số lẻ, $\beta=2$ : số chẵn, số $n \in \beta=1$ là số lẻ, số $n \in \beta=2$ là số chã̃n, các hệ số điều hòa cầu $\overline{\bar{C}}_{n, m}, \overline{\bar{S}}_{n, m}$ được xác định như sau:

$$
\begin{gathered}
m=0, n \in \beta=\left\{\begin{array}{l}
1 \quad \overline{\bar{C}}_{n, m}=\bar{C}_{n, m} \\
2 \overline{\bar{C}}_{n, m}=\bar{C}_{n, m}+\frac{G \cdot M_{0}}{G \cdot M} j_{n}^{(0)}
\end{array}, \overline{\bar{S}}_{n, m}=0,\right. \\
m \geq 1 \quad \overline{\bar{C}}_{n, m}=\bar{C}_{n, m}, \overline{\bar{S}}_{n, m}=\bar{S}_{n, m},
\end{gathered}
$$

$\bar{C}_{n, m}, \bar{S}_{n, m}$ là các hệ số điều hòa cầu được chuẩn hóa của mô hình trọng trường Quả đất EGM, các hệ số điều hòa vùng chẵn

$$
\begin{gathered}
j_{2}^{(0)}=0,108262982131 \cdot 10^{-2}, j_{4}^{(0)}=-0,237091120053.10^{-5} ; \\
j_{6}^{(0)}=0,608346498882 \cdot 10^{-8}, j_{8}^{(0)}=-0,14268108792 \cdot 10^{-10}, j_{10}^{(0)}=0,121439275882.10^{-13},
\end{gathered}
$$

còn các hệ số $j_{n}^{(0)}$ với $\mathrm{n}$ là số chẵn lớn hơn 12 đều bằng 0 .

Chúng ta đã thấy rằng cùng với các dữ liệu đo trọng lực chi tiết, các dữ liệu độ cao chuẩn và độ cao trắc địa trên các điểm độ cao quốc gia thuộc tập hợp các điểm cơ sở hạng $B$ cho phép giải quyết bài toán hiệu chỉnh các hệ số khai triển điều hòa của mô hình trọng trường Trái đất EGM2008 cho phù hợp với trọng trường trên lãnh thổ quốc gia. Khi đó, mô hình trọng trường Trái đất EGM2008 được hiệu chỉnh cho phép không chỉ nhận được các giá trị dị thường độ cao độ chính xác cao tương ứng với mô hình quasigeoid quốc gia cuối cùng, mà còn nhận được các giá trị vật lý khác như dị thường trọng lực Faye, các thành phần độ lệch dây dọi sát với các giá trị thực của chúng tại các điểm trên lãnh thổ quốc gia. 


\section{Kết luận}

Trong trường hợp sử dụng mô hình quasigeoid quốc gia khởi đầu được xây dựng theo công thức (1), trên các điểm độ cao hạng I, II quốc gia có đo GPS và xử lý các dữ liệu GPS trong ITRF chúng ta sẽ xác định được tập hợp các điểm cơ sở hạng $B$ mà các giá trị tuyệt đối của các độ chênh giữa dị thường độ cao GPS/thủy chuẩn và dị thường độ cao trọng lực cục bộ nhỏ hơn $10 \mathrm{~cm}$. Các điểm này sẽ cho phép xây dựng mô hình quasigeoid quốc gia cuối cùng với độ chính xác ở mức $\pm 2 \mathrm{~cm}$ sau khi khớp các giá trị dị thường GPS/thủy chuẩn với các giá trị dị thường độ cao trọng lực cục bộ theo phương pháp collocation trung phương.

Ngoài ra, bài báo khoa học này còn đề xuất bài toán hiệu chỉnh các hệ số khai triển điều hòa của mô hình trọng trường Trái đất EGM2008 cho phù hợp với trọng trường trên lãnh thổ quốc gia trên cơ sở sử dụng các dữ liệu trọng lực chi tiết và các dữ liệu độ cao chuẩn, độ cao trắc địa trên các điểm cơ sở hạng $\mathrm{B}$. Điều này cho phép không chỉ nhận được các giá trị dị thường độ cao độ chính xác cao tương ứng với mô hình quasigeoid quốc gia cuối cùng, mà còn nhận được các giá trị vật lý khác như các giá trị dị thường trọng lực Faye, các thành phần độ lệch dây dọi sát với các giá trị thực của chúng tại các điểm trên lãnh thổ quốc gia.

Tác giả cám ơn Chương trình KH\&CN trọng điểm cấp Nhà nước KC-09/11-15 "Nghiên cứu Khoa học và Công nghệ phục vụ quản lý biển, hải đảo và phát triển kinh tế biển" đã hỗ trợ nội dung bài báo này. $O$

\section{Tài liệu tham khảo}

[1]. Forsberg R., Strykowski G., lliffe J.C., Ziebart M., Cross P.A., Tscherning C.C., Cruddace P. (2001). OSGM02: A new geoid model of the British Isles. www.discovery.ucl.ac.uk>.. >UCL.DISCOVERY.

[2]. Hà Minh Hòa (2012). Nghiên cứu xác định thế năng trọng trường thực $W_{0}$ của mặt Geoid cục bộ trùng với mặt biển trung bình tại trạm nghiệm triều Hòn Dấu. Báo cáo khoa học. Tuyển tập báo cáo Hội nghị Khoa học và Công nghệ "Trắc địa và Bản đồ vì sự nghiệp tài nguyên và Môi trường". Viện Khoa học Đo đạc và Bản đồ - Hội Trắc địa, Bản đồ và Viễn thám Việt Nam. Hà Nội - Tháng 10/2012. Trg. 6-19.

[3]. Hà Minh Hòa (2013a). Các vấn đề liên quan đến việc xây dựng hệ quy chiếu không gian quốc gia. Tạp chí Khoa học Đo đạc và Bản đồ, No18, 12/2013, trg. 1-10.

[4]. Ha Minh Hoa (2013b). Estimating the geopotential value $W_{0}$ of the local geoid based on data from local and global normal heights of GPS/Leveling points in Vietnam. Geodesy and Cartography. Taylor \& Francis. UDK 528.21, doi:10.3846/20296991.2013.823705, V.39 (3): 99-105.

[5]. Hà Minh Hòa (2014a). Hiệu chỉnh các hệ số điều hòa cầu của mô hình trọng trường Quả đất nhờ các kết quả bình sai mạng lưới độ cao hạng I, II quốc gia trong hệ độ cao dựa trên mặt Geoid cục bộ. Tạp chí Khoa học Đo đạc và Bản đồ, No19, 03/2014, trg. 1-8.

[6]. Hà Minh Hòa (2014b). Lý thuyết và thực tiễn của Trọng lực trắc địa. NXB Khoa học và Kỹ thuật, 592 trg., Hà Nội - 2014.

[7]. Hà Minh Hòa (2014c). Đánh giá độ chính xác của mô hình Quasigeoid EGM2008 
trên lãnh thổ Việt Nam. Tạp chí Khoa học Đo đạc và Bản đồ, No20, 06/2014, trg. 1-13.

[8]. Hà Minh Hòa (2014d). Phương pháp chuyển độ cao chuẩn nhà nước lên các mặt biển trung bình tại các trạm nghiệm triều trên các đảo xa. Báo cáo khoa học. Kỷ yếu Hội nghị Khoa học "Trắc địa và Bản đồ vì hội nhập quốc tế" ngày 08/07/2014. Viện Khoa học Đo đạc và Bản đồ, Hội Trắc địa, Bản đồ và Viễn thám Việt Nam, trg. 2-15.

[9]. Hà Minh Hòa, Nguyễn Tuấn Anh (2014e). Nghiên cứu khả năng hiệu chỉnh các hệ số khai triển điều hòa cầu của thế trọng trường Quả đất của mô hình EGM2008 dựa trên các dữ liệu đo trọng lực chi tiết ở Việt Nam. Báo cáo khoa học. Kỷ yếu Hội nghị Khoa học "Trắc địa và Bản đồ vì hội nhập quốc tế" ngày 08/07/2014. Viện Khoa học Đo đạc và Bản đồ, Hội Trắc địa, Bản đồ và Viễn thám Việt Nam, trg. 21-37.

[10]. Hà Minh Hòa (2014f). Tiếp cận phương pháp hoàn thiện và hiện đại hóa hệ độ cao quốc gia. Tạp chí Khoa học Đo đạc và Bản đồ, No21, 09/2014, trg. 1-12.

[11]. Hirt C., Featherstone W. E., Marti U. (2010). Combining EGM2008 and SRTM/DTM2006.0 residual terrain model data to improve quasigeoid computations in mountainous areas devoid of gravity data. Journal of Geodesy, Volume 84, Number 9, 557567, DOI: 10.1007/s00190-010-0395-1.

[12]. Iliffe J.C., Ziebart M., Cross P.A., Forsberg R., Strykowski G., Tscherning C.C. (2003). OSGM02: A New model for converting GPS-derived heights to local height datums in Great Britain and Ireland.

[13]. Veronneau M., Huang J. (2007). Moving to a Modernized Height Reference System in Canada: Rationale, Status and Plans. Natural Resources Canada. American Geophysical Union, Spring Meeting 2007. 1214 Geopotential theory and determination (0903), 1229 Reference systems. 007AGUSM.G33B.05V.

[14]. Véronneau M. (2014). The Canadian Geodetic Vertical Datum of 2013. Canadian Institute of Geomatics, Ottawa Branch, 29 April 2014, Natural Resources Canada, 33 p.O

\section{Summary}

Problem of fitting of local gravimetric and GPS/leveling height anomalies in task of modernization of the national vertical system

Assoc. Prof. Dr. Sc. Ha Minh Hoa

Vietnam Institute of Geodesy and Cartography

This scientific article determined base for selection of first and second order height points with absolute values of defferences between local gravimetric and GPS/leveling height anomalies less $10 \mathrm{~cm}$ that are used for construction of the last national quasigeoid based on fitting of the GPS/leveling height anomalies and local gravimetric height anomalies by the collocation method. Besides, this article still proposed method of fitting of abovementioned values by correction of the spherical harmonic coefficients of the EGM2008 based on the disturbing potentials at the B order base points. $O$

Ngày nhận bài: 17/11/2014. 